\title{
A Brief Review on the Relationship Between Pain and Sociology
}

\author{
Nader Nader ${ }^{1}$, Haleh Farzin ${ }^{2}$ and Hanieh Sakha ${ }^{3, *}$ \\ ${ }^{1}$ Department of Anesthesiology, University at Buffalo, Buffalo, USA \\ ${ }^{2}$ Tabriz University of Medical Sciences, Tabriz, Iran \\ ${ }^{3}$ Sociology Department, Tehran North Branch, Islamic Azad University, Tehran, Iran \\ "Corresponding author: Sociology Department, Tehran North Branch, Islamic Azad University, Tehran, Iran. Email: h.dsakha@yahoo.com \\ Received 2019 October 30; Revised 2020 March 01; Accepted 2020 March 21.
}

Keywords: Pain, Medical Sociology, Sociology

\section{Dear Editor,}

Throughout history, pain theories have been supposed by biomedicine, especially regarding its diagnosis and treatment aspects. Therefore, the feeling of pain is not only personal experience and is affected by social background; therefore, it involves extensive systems of signals.

The challenges in emotional and sentimental dimensions of pain originate from scientific medicine (i.e., the dominant theory is also referred to as the specificity theory); however, this theory has accepted some alterations by emerging physiology. Then, Von Frey suggested the theory of cutaneous senses (i.e., Muller's concept: the common sensation of combined four major skin receptors leading to a proper sensation), 50 years after the specificity theory. The pain pathway was composed of spinothalamic tracts and thalamus with an inhibitory effect of cortex. Pain is referred to as a series of unique experiences with various reasons and qualities. Despite the gate control theory, the biological aspect overcomes the social aspect. Vrancken provided a more extensive definition of pain and found five approaches: The somatico-technical, dualistic bodyoriented, behaviorist, phenomenological, and consciousness approaches.

The Westem model combined physical, emotional, and existential aspects of the human body. On the other hand, Kotarba felt confused about the basic origins of chronic pain. Freund demonstrated and argued with Durkhemian about the sociological approach to emotions. Lynch provided a piece of evidence about the correlation between cardiovascular disease and emotionally life-threatening occurrences. Helman supposed a distinction between private and public pain.

According to Turner, the consideration of the emotional aspect of pain could lead to effective, emotional, and social responses to pain. On the contrary, the theory of em- bodiment is based on the sociology view of health and illness.

Based on the medicalized phenomenon, pain is an objective experience. Therefore, the sociological conceptualization of the chronic disease needs understanding the social meaning (i.e., body acceptance). It seems that neglecting the "living body" by medical sociologists is associated with a global weak concentration on normal daily experiences of the body. The real importance of social fundamentals of human experiences about the disease and various negative social pressures in such conditions define the language for the expression of conditions and self-serving methods (1).

However, sociological stories about chronic diseases neither could deny the body's existence nor were considered as a clear truth. The social aspect originates from social meanings, such as the child's appearance and behavior, and the reduced ability of the infant to induce effective responses in these conditions. The crisis and negotiation models were developed by Gerhardt. In the negotiation model, some diseases, such as colitis and emphysema, are not referred to as particular unusual events and do not cause remarkable effects on interactions. These conditions have caused some problems and resulted in some social responses in patients. The bridge between biological and social facts is an outcome of these interactions (2).

The administration of painkillers is an essential approach to inhibit the self-management of pain. Cicely stated that "much of our total pain experience is composed of our mental reaction..."'. This shows the integrity of body and personality (3).

Professional sport and pain have made a special space for interrelations between sports medicines, body, and culture, which has resulted in historical alterations in sports medicine. Anthropology is the main bases of ethnography. 
In this method, we could record pain in a sports context. However, the basic source of pain has generally been forgotten (4).

If acute pain is developed into chronic pain, therefore, it will be an individualized or semi-individualized experience. The athletes may respond to pain by thought suppression, depression, and suicidal thoughts. In high-level competitive sports, athletes try to ignore pain (5).

The social epidemiology shows an imbalanced distribution of health, illness, and disability among various social groups. The social support and socio-cultural level can result in several types of pain. It means the status of athletes might define their pain experiences. Gender is one of the important contributing factors affecting the type of pain (i.e., females are more likely to seek health services for pain relief).

According to melody Fairchild, coaches are the founder of pain relief and reduce the intensity of injuries (6).

Chronic non-cancer pain (CNCP) has become a serious public health issue affecting more than 70 million people globally (7).

CNCP is a serious public health issue, which is caused by the lack of awareness about chronic pain management among the general population.

Key informants from Karachi and Kuwait also mentioned, "lack of awareness about chronic pain management among the general population". And "society is immature for pain specialty" (8).

\section{Footnotes}

Authors' Contribution: None declared by author. Conflict of Interests: The authors declared no conflicts of interest.

Funding/Support: This study was not financially supported by any organization.

\section{References}

1. Bendelow GA, Williams SJ. Transcending the dualisms: Towards a sociology of pain. Sociol Health Illness. 1995;17(2):139-65.

2. Kelly MP, Field D. Medical sociology, chronic illness and the body. Sociol Health Illness. 1996;18(2):241-57. doi: 10.1111/1467-9566.ep10934993.

3. Clark D. Total pain', disciplinary power and the body in the work of Cicely Saunders, 1958-1967. Soc Sci Med.1999;49(6):727-36.

4. Howe PD. Sport, professionalism, and pain: Ethnographies of injury and risk. Psychology Press; 2004.

5. Konietzny K, Chehadi O, Levenig C, Kellmann M, Kleinert J, Mierswa T, et al. Depression and suicidal ideation in high-performance athletes suffering from low back pain: The role of stress and painrelated thought suppression. Eur J Pain. 2019;23(6):1196-208. doi 10.1002/ejp.1387. [PubMed: 30821880].

6. Nixon HL. Explaining pain and injury attitudes and experiences in sport in terms of gender, race, and sports status factors. J Sport Soc Issues. 2016;20(1):33-44. doi: 10.1177/019372396020001004.

7. Jackson TP, Stabile VS, McQueen KAK. The global burden of chronic pain. ASA Newsletter. 2014;78(6):24-7.

8. Lakha SF, Ballantyne P, Badr H, Agboatwala M, Mailis A, Pennefather P. Perspective of pain clinicians in three global cities on local barriers to providing care for chronic noncancer pain patients. Pain Res Manag. 2019;2019:3091309. doi: 10.1155/2019/3091309. [PubMed: 30863473] [PubMed Central: PMC6377973]. 